\title{
Procesos biográficos de la modernización en Chile
}

\author{
Felipe Ghiardo Soto
}

Centro de Estudios Sociales cidpa, Valparaíso, Chile.

Email: felipe@cidpa.cl

\section{Oscar Dávila León}

Centro de Estudios Sociales cIDPA, Valparaíso, Chile.

Email: oscar@cidpa.cl.

Resumen $^{1}$ : El artículo presenta un conjunto de tendencias sociodemográficas a partir de las cuales se propone una lectura de los cambios en los cursos de vida en Chile. Tomando como referencia el período 1950-2010, se aplicó un análisis de cohortes que recorre los procesos de escolarización de distintas generaciones, el ingreso al campo laboral, las tendencias en la formación de pareja y la procreación.

Palabras clave: curso de vida, edad, cohortes.

\section{Biographical processes of modernization in Chile}

Abstract: The paper presents a set of demographic trends from which is proposed a reading of the changes in the courses of life in Chile. Taking by reference the period 1950-2010, was applied a cohort analysis that went through schooling processes of different generations, the access to work and the trends in family formation and childbearing.

Key words: course of life, age,cohort

\section{Processos biográficos da modernização no Chile}

Resumo: O artículo apresenta um conjunto de tendências sócio demográficas a partir das quais se propõe uma leitura das mudanças nos cursos de vida no Chile. Tomando como referencia o período 1950-2010, aplicou-se uma análise de grupos de pessoas que percorre os processos de escolarização de diversas gerações, o ingresso ao campo laboral, as tendências na formação dos casais e a procriação.

Palavras-chave: curso de vida, idade, grupos de pessoas.

$$
* * *
$$

\section{Introducción}

En un artículo que trata sobre los cambios del Chile contemporáneo, Pedro Güell plantea que una de las particularidades del período actual es que los cambios aceleran y «cambian de escala» (Güell, 2007). Si hasta hace 
poco las fuerzas del cambio «bajaban» desde las instituciones a la sociedad civil, en las últimas dos décadas se produjo un bucle que estaría complejizando e incluso invirtiendo la dirección del flujo que gobierna los cambios. Del dominio de la «lógica de las instituciones» se pasaría a un tipo de sociedad en que las prácticas de los sujetos se estarían tomando el motor de las transformaciones societales (Güell, Frei y Palestini, 2009). Y lo que nos interesa es que uno de los resortes de este giro son los trabajos biográficos que supone el proceso de individuación. Si «en las expectativas y temores de los individuos, los procesos de construcción de sus proyectos biográficos y sus relaciones primarias en la vida cotidiana» (Güell, 2007: 8) es donde cabe buscar el germen de los movimientos que hoy interpelan a las instituciones, sugiere que los procesos biográficos pueden ser un analizador que revela las contradicciones de una sociedad y abre una vía de acceso para comprender los procesos de cambio social.

Es lo que hizo Ulrich Beck cuando escribió sobre la sociedad del riesgo y la necesidad de una modernidad reflexiva. Todo el apartado sobre las paradojas de la individualización bajo el régimen del Estado Benefactor se sostiene en una reflexión sobre las nuevas formas de producción de biografías que emergen en el escenario de la Alemania Federal de postguerra. Su tesis central es que si por un lado todo el sistema de prestaciones que articuló el Estado de Bienestar logró mejorar las condiciones de vida del conjunto — «efecto ascensor»— y separar el destino individual de las dependencias familiares y de clase, ese mismo proceso habría presionado cada vez más a una gestión individual de las incertidumbres y el riesgo que amarró las posibilidades de acceder al mercado de trabajo y consumo a los recursos que pudieran entregar el sistema escolar y el de protección social. El resultado es una individualización ambivalente que termina por estandarizar e institucionalizar los modelos biográficos (Beck, 1998).

La tesis de la institucionalización de las biografías, o de las biografías como nuevas instituciones, ha dado pie a todo un debate reciente sobre su permanencia o su disolución (Kholi, 2005, 2007). La cuestión es si se puede hacer esa lectura en que esas condiciones institucionales no se dieron con la misma intensidad ni de la misma forma. En Chile, en particular, los intentos de modernización impulsados por el Estado alcanzaron a formar un sistema de instituciones análogo a los del Estado de Bienestar europeo, pero ni la estructura del sistema productivo llegó a ser industrial, ni las adscripciones «tradicionales» desaparecieron, ni los sistemas de protección alcanzaron a consolidarse, y cuando avanzaban hacia esa vía, se impuso un giro neoliberal radical que ha marcado los veinte años de «transición a la democracia» y el modo de inserción en el mundo globalizado. De ahí la pertinencia de ver qué pueden decir los procesos biográficos sobre estas transformaciones, si aplican también acá las paradojas de la individualización, la tesis de la institucionalización de las biografías y la disolución del efecto de clase.

Esas son algunas de las cuestiones que orientan la investigación de la que forma parte este texto. En lo medular, el estudio intenta establecer una 
lectura que vincule las trayectorias de vida de distintas generaciones, ver qué tendencias describen y hacia dónde apuntan, con los procesos sociohistóricos de la «modernización» en Chile asumiendo, de paso, uno de los retos que se viene planteando la demografía para su propia renovación: pasar de estudios sobre la transición demográfica y el constructo «población» a un análisis sobre las estructuras demográficas de la diferenciación social (Canales, 2004).

\section{Biografías y cambio social: enfoques investigativos}

Existe una serie de enfoques teórico-metodológicos que trabajan sobre este plexo estructuras-biografías-tiempo-histórico que en el último tiempo se vienen aplicando con cierta regularidad a temáticas de demografía social. Enfoques como el Biografía-sociedad que desarrolla Lüdger Pries (Pries, 1996), el Enfoque Biográfico (Bertaux, 1999; De Coninck y Godard, 1998), el de Historias de vida (Thompson, 1993; Ferrarotti, 2011), el análisis que hace Bourdieu sobre las trayectorias sociales (Bourdieu, 1988) o el enfoque de los Cursos de vida que iniciara Glen Elder (Blanco, 2010; Sepúlveda, 2010), entre otros, han prestado herramientas que vinieron a renovar el andamiaje teórico y los recursos investigativos en este campo. Temas habituales como los eventos de transición se abordan ahora como parte de trayectorias o cursos de vida que ocurren en un contexto histórico, institucional, social que los determina, sin que ello quite espacio para la variedad de motivos, decisiones y contingencias que suponen la subjetividad y la vida práctica.

Con esta óptica se vienen produciendo numerosos estudios sobre trayectorias escolares (Raczinski, 2001; ), formas de transición hacia la vida adulta (Casal et al. 2006; Mora y Oliveira, 2009; Miret, 2005), sobre el tránsito de los estudios al trabajo (Jacinto, 2006; Pérez-Baleón, 2012), trayectorias laborales de distintos grupos y generaciones (Henríquez y Uribe-Echeverría, 2003; Longo, 2010; Schkolnik, 2006), su relación con las trayectorias familiares y las prácticas reproductivas (Oliveira y García, 1990; Quilodrán y Juárez, 2009), entre otros temas que ilustran la variedad de aristas por cubrir y de apuestas metodológicas por explorar. Una vista panorámica presenta estudios cuantitativos que se basan en fuentes secundarias para comparar entre grupos o seguir cohortes a través del tiempo (Pacheco y Blanco, 2005; Davia, 2013); otros que elaboran sus propias encuestas con una lógica retrospectiva o longitudinal (Longo, 2010). También se pueden encontrar muchos trabajos que profundizan la dimensión cualitativa mediante entrevistas en profundidad y otras técnicas conversacionales (Oliveira, y García, 1990; Scoppetta, 2009; Guichard, et al., 2013; Roberti, 2012), o estrategias mixtas que mezclan técnicas cuantitativas y cualitativas en una misma investigación (Blanco y Pacheco, 2003; Longo, 2010; Mora y Oliveira, 2009.).

En nuestro caso, el diseño original del estudio del que forma parte este texto ensaya ambos registros, el cualitativo mediante entrevistas y 
grupos de discusión y el cuantitativo mediante el análisis de diversas fuentes de datos estadísticos. En lo que sigue presentamos resultados de este segundo momento. Aquí el propósito no es más que trazar «paisajes de época» y descubrir tendencias generales. Para eso nos basamos en dos series de fuentes estadísticas: la de los censos de población desde 1930 a 2002, y la de Estadísticas Vitales que entrega el Instituto Nacional de Estadísticas (INE) desde 1952 hasta el 2010. De los censos extrajimos datos sobre niveles de escolaridad, participación en la población económicamente activa (PEA) y estado civil. Y de las estadísticas vitales, datos sobre fecundidad y nupcialidad. Con eso hicimos un análisis de cohortes y ensayamos dos ejercicios que organizan la exposición. ${ }^{2}$ En el primero comparamos la situación de distintas cohortes a una misma edad; y en el segundo seguimos un mismo indicador en distintos momentos de una misma cohorte. Para ambos ejercicios partimos desde el momento en que cada cohorte tuvo entre 15 y 19 años, básicamente porque lo que nos interesaba era partir el análisis desde que se empiezan a producir los principales eventos de transición demográficos. Los resultados se exponen a continuación.

\section{La producción social de las edades}

La pregunta que recorre esta sección es ver cómo y en qué han cambiado los regímenes de transición en las últimas seis a siete décadas. ${ }^{3}$ Nos concentramos en la escolarización, el ingreso al mundo laboral y algunos aspectos vinculados a la conformación de pareja y familia. Con esos datos realizamos un análisis diacrónico - entre cohortes - para ver cómo varían los niveles de un mismo indicador a determinada edad cronológica, y un análisis sincrónico del mismo indicador entre subconjuntos de una misma cohorte, como un acercamiento general a las variaciones estructurales en los modos de generación del período. ${ }^{4}$

\section{a) Años de estudio: más con más}

Partimos por una cuestión básica: cuántos, quiénes y por cuánto tiempo vivieron la experiencia de la escolarización. Como referencia utilizamos la tasa de participación en la Enseñanza Secundaria a los 15-19 años de cada cohorte, que en general coincide con las edades que cubre este ciclo. Las tendencias se exponen en el gráfico 1. Cuando empiezan las curvas (1952), la Ley de Instrucción Primaria Obligatoria (1920) llevaba treinta años tratando de «forzar» la escolarización del bajo pueblo. ${ }^{5}$ Su efecto se nota en que cerca del $70 \%$ de los niños y niñas que integran la primera de estas cohortes pasó por el ciclo básico. Pero la mayoría llegó hasta ahí. Menos de una quinta parte de las primeras dos cohortes tuvo estudios secundarios. No eran todavía prioridad ni para las familias ni para el Estado. Lo importante, en ese entonces, era la alfabetización, primer paso y piso en la ruta hacia la «modernización cultural» de una sociedad todavía agraria y de transmisión oral. ${ }^{6}$ Por ahí se entiende que la diferencia clave fuera urbano/rural y no hombre/mujer. Ese es el primer punto. La cuestión era de infraestructura: no había Liceos rurales; ${ }^{7}$ pero también de «mundos»: la ruralidad todavía 
era distancia física y «cultural» que sólo se reducía con un «cambio de mundo» que pocos hicieron.

El segundo punto es que si bien los estudiantes secundarios se concentraban en las ciudades, en 1950, 1960, 1970, 1982 inclusive, estaban lejos de ser la realidad común entre los urbanos de las respectivas cohortes. La diferencia remite a la formación de clases: la secundaria era un espacio al que accedían jóvenes de las élites y los sectores medios de las ciudades que buscaban asegurar su posición mediante cargos en el Estado o las empresas. El resto, la mayoría, se ajustaba a estrategias de reproducción ajenas a la escuela y los títulos. ${ }^{8}$

Desde los años cincuenta, sin embargo, se empieza a notar una inclinación ascendente en las curvas que coincide con las transformaciones estructurales de los años del desarrollismo. Producto de la expansión del Estado, la complejización de los procesos productivos, el crecimiento de las ciudades y las expectativas de movilidad para técnicos y profesionales, nuevos segmentos empiezan a trasladar sus estrategias hacia la vía escolarizante. Su expresión política fue la Reforma Educacional de Frei Montalva (1965), que edificó liceos y amplió la infraestructura para «democratizar» el acceso a la educación secundaria, técnica y superior. Primero fue en las ciudades: entre 1950 y 1982 el quiebre se restringe a hombres y mujeres urbanos de clase media y algunos sectores asalariados; ${ }^{9}$ pero desde los ochenta y sobre todo a partir de los años noventa, las tendencias llegan a los sectores populares urbanos y a las zonas rurales con una fuerza tal que en 2011 el porcentaje alcanza el nivel de las ciudades.

\section{Gráfico 1: porcentaje con estudios secundarios según sexo por área de residencia. Tramo 15 a 19 años. Chile 1952-2011}

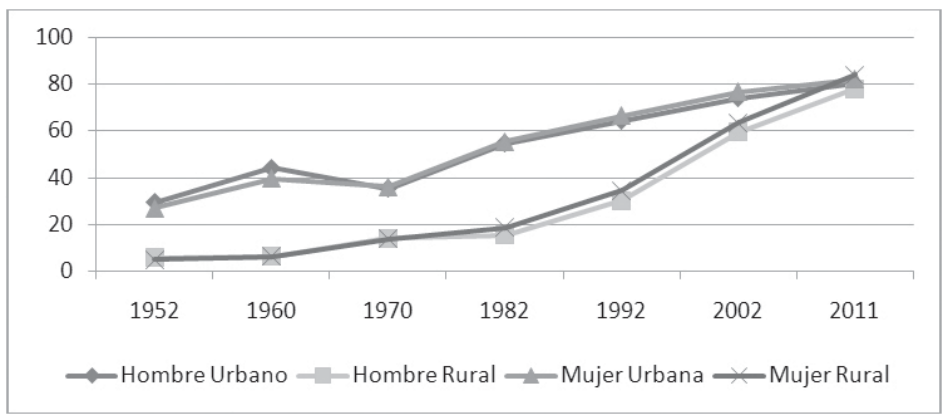

Fuente: Elaboración propia sobre la base de serie de censos de población 1952-2002, CASEN 2011. 
Entre extremo y extremo del gráfico, la situación se ha invertido y homogenizado. En las cohortes actuales, cerca de 8 de cada 10 jóvenes entre 15 a 19 años tiene estudios secundarios, y los demás, o todavía no han terminado la básica —en torno al $12 \%$ — o ya están en la educación superior - $8 \%$ entre los urbanos y $2 \%$ entre los rurales-. Para esta suerte de institucionalización de esta fase biográfica se han debido cruzar una variedad de procesos de distinto orden que van desde la inversión pública y privada en establecimientos escolares y una serie de programas ministeriales para elevar los años de escolaridad, ${ }^{10}$ hasta las transformaciones de los territorios, ${ }^{11}$ las exigencias de las empresas, el peso de un discurso que asocia movilidad y desarrollo con años de escolaridad y que alienta a nuevas segmentos cada vez más amplios a invertir en años de estudio como eje de las estrategias de reproducción. El límite de este proceso es que la escolaridad breve que caracterizó a las generaciones hoy adultas de sectores populares constituye hoy un «factor» que explica la desigualdad en las condiciones de «educabilidad» de las generaciones actuales y que el sistema escolar absorbe y reproduce en su estructura "conscientemente segmentada» en función de las clases sociales (cf. OCDE, 2004). ${ }^{12}$

\section{b) La reducción del trabajo adolescente}

El sentido de este cambio en los modos de generación se entiende mejor cuando se aborda la participación en la fuerza de trabajo. El gráfico 2 lo que demuestra es que si a mediados de siglo la vida para la mayor parte de los hombres urbanos y rurales a los 15-19 años no estaba en el Liceo, era porque ya contaban como parte de la Población Económicamente Activa (PEA) - 60\% en las ciudades y 80\% en las zonas rurales-. Su explicación es estructural e histórica: cuando empiezan las curvas, lo rural-agrario-tradicional es todavía gravitante en la composición de la producción y el habitar en Chile. Que los muchachos trabajaran desde edades tempranas formaba parte de las necesidades de la economía familiar campesina e indígena y de las obligaciones que exigía el latifundio por el arriendo de tierras al inquilinaje. No extraña, en ese sentido, que del total de oficios de la época, el de Peón Agrícola fuera el más habitual entre los varones menores de 20 años (DGE, 1930-1960). Tampoco la frecuencia que tuvo el trabajo en las ciudades. Fue uno de los recursos habituales de las primeras industrias. Niños y muchachos trabajadores los hubo en la industria de alimentos, la metalurgia, el vidrio. Lo hacían por necesidad, por ayudar a sus madres, pero también porque se asumía como «normal» o incluso «bueno» que los niños trabajaran. En eso coincidían sectores empresarios y obreros: trabajar desde pequeños «forjaba el carácter» y aseguraba la continuidad del beneficio industrial o de la familia y la clase (Rojas, 1996). Por eso al principio tuvieron poco efecto las normas que buscaron limitar el trabajo infantil. ${ }^{13}$ En la práctica siguieron trabajando en la agricultura, las minas y la industria, al punto que hasta al menos la década de 1960 aparece como la situación más frecuente entre los menores de 20 años. ${ }^{14}$

Eso en el caso de los hombres. Entre las mujeres la participación temprana en la PEA fue bastante más baja. A los 15 ó 19 años lo más frecuen- 
te era una inactividad por quehaceres del hogar, aunque no deja de ser relevante la cantidad de mujeres que trabajaban en las ciudades, muchas de ellas probablemente jóvenes que llegaban de los campos a enrolarse en el servicio doméstico o puestos del comercio en busca de una vía para cambiar una suerte que en su defecto significaba mantenerse en la condición de familiar no remunerado o servidumbre (DGE, 1930; 1940; 1952).

\section{Gráfico 2: tasa de participación en la pea según sexo por área de residencia. Tramo 15 a 19 años. Chile 1952-2011}

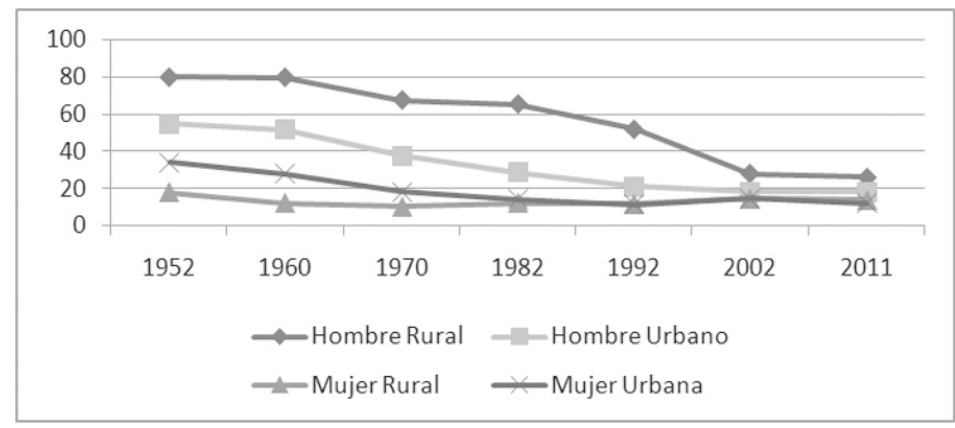

uente: Elaboración propia sobre la base de serie de censos de población 1952-2002, CASEN 2011.

La primera inflexión importante tanto para hombres como para mujeres se produjo entre 1960 y 1970. A partir de ahí, el trabajo empieza a perder frecuencia, primero en las ciudades, y después, desde la década de los ochenta, en las zonas rurales. El resultado es que al final de este período la situación también se ha invertido y estandarizado: el trabajo deja de ser el mundo de referencia para los jóvenes a los 15, 16 ó 17 años, sea donde sea que vivan. Cambió el régimen de discurso: ya no es «deseable» o incluso «normal» que a esta edad trabajen; lo que se espera y aspira es que puedan estudiar. Es lo que aparece al superponer los gráficos 1 y 2: todo lo que baja la participación en la PEA masculina y la inactividad femenina, sube en matrícula de enseñanza media. Y fue ese movimiento bascular el que convirtió a esta en una edad para los estudios y ya no de trabajo, preparando, de esa forma, las condiciones para la emergencia y expansión de la «adolescencia» y «juventud» en Chile. ${ }^{15}$

\section{c) Tránsito estudios-trabajo: el «efecto dominó» y sus límites}

Lo relevante de este doble movimiento que desplaza el ingreso definitivo al mundo laboral es que activa un «efecto dominó» que alcanza a las edades posteriores. Es lo que se observa en el gráfico 3: si bien el descenso 
más notorio en la proporción de hombres laboralmente activos se dio justamente entre los 15 a 19 años, en el tramo 20 a 24 años se anota un movimiento menos intenso pero en la misma dirección.

\section{Gráfico 3: tasa de participación pea masculina según tramo etario. Chile, 1952-2011}

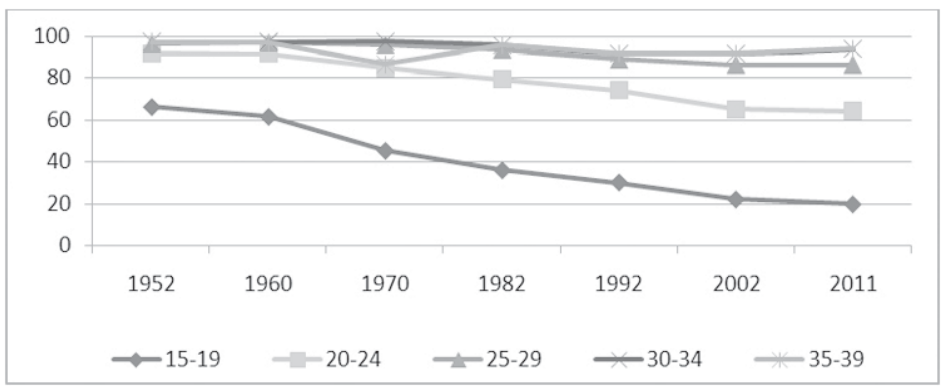

Fuente: Elaboración propia sobre la base de serie de censos de población 1952-2002, CASEN 2011

El descenso es lineal, aunque tiene dos momentos de inflexión: el primero en la década de los sesenta y el segundo en los noventa, y parecieran coincidir con los períodos de mayor crecimiento en la matrícula de educación superior. Como se observa en el gráfico 4, si durante toda la primera mitad del siglo xx los estudiantes de educación superior fueron una minoría marginal — del $2 \%$ al 3\%—, reducida nada más que a un segmento de los sectores que invertían en títulos profesionales, la presión por acceso y democratización que parte en los sesenta y la más reciente inflación en la oferta y demanda de títulos técnicos y universitarios está implicando que en este tramo también se registre ese movimiento bascular desde el trabajo a los estudios que incluso alcanza, aunque debilitado, al tramo entre 25 y 29 años. 


\section{Gráfico 4: porcentaje con estudios universitarios según sexo. Tramo 20 a 24 años. Chile, 1952-2011}

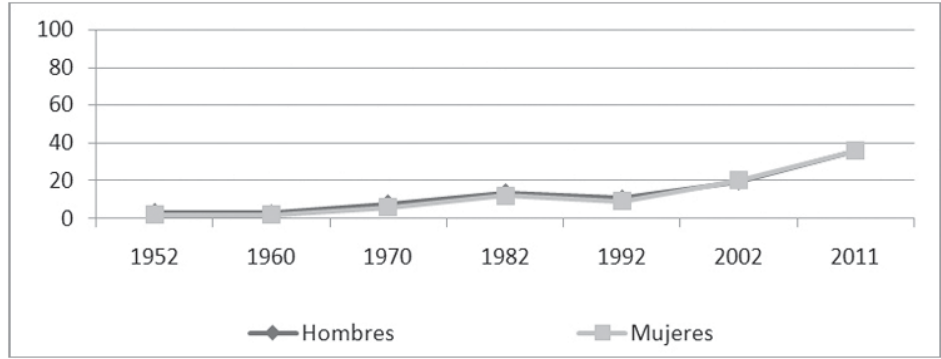

Fuente: Elaboración propia sobre la base de serie de censos de población 1952-2002, CASEN 2011.

La tendencia expresa un nuevo cambio en los modos de generación particularmente significativo en algunos segmentos medios y medio-bajos, que apuestan por una vía escolarizante de largo aliento ausente en la trayectoria de anteriores generaciones. Y lo interesante es que las mujeres participan a la par de los hombres, con porcentajes que dibujan las mismas curvas y al mismo nivel, activando un efecto de ondas que en las edades siguientes eleva — en vez de bajar, como en los hombres - la participación femenina en la PEA (gráfico 5). El cambio es notable: si entre 1952-1992 sólo un tercio de las mujeres entre 25 y 34 años estaba laboralmente activa, entre 1992 y 2011 el porcentaje por poco se duplica. Bordeando los 30 años las mujeres no se «desactivan» como antes; ahora apuestan por su independencia y permanecen en actividad, sea por estudios, por trabajo o por una combinación de ambos, creando, en ese trance, las condiciones para una nueva «condición femenina» y hasta una nueva «edad», la de «adultasjóvenes», con «temas» y prácticas propias. ${ }^{16}$

\section{Gráfico 5: tasa de participación pea femenina según tramo etario. Chile, 1952-2011}

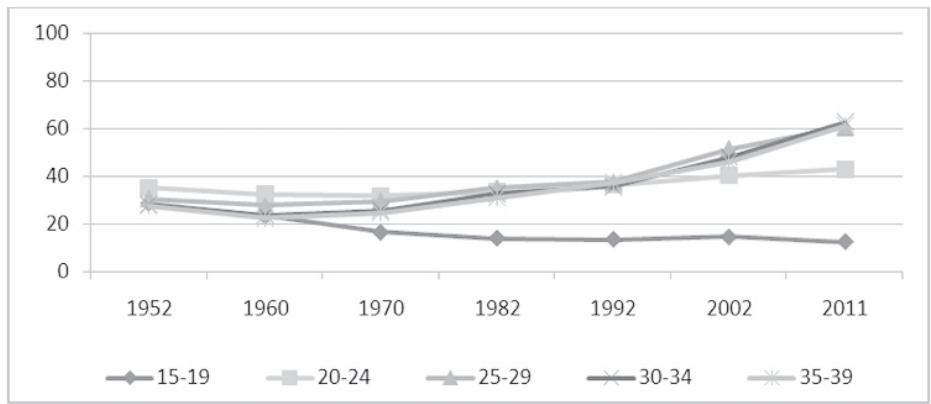

Fuente: Elaboración propia sobre la base de serie de censos de población 1952-2002, CASEN 2011. 
Lo que nos interesa destacar es que si por un lado esta prolongación de los años de estudio ha logrado homologar un tipo de trayectoria para el conjunto de las actuales generaciones adolescentes —hasta los 18 ó 19 años, casi todos estudian-, que a su vez desplaza el tránsito estudiostrabajo y reduce el peso de la dicotomía trabajadores/estudiantes, ese efecto dura lo que dura la secundaria. ${ }^{17}$ Sólo en los sectores con mayores ingresos se da una continuidad estable y mayoritaria entre el término de la enseñanza media y el comienzo de los estudios superiores, en su mayoría universitarios, pero ya al pasar a los sectores medio y medio-bajo, ese mismo punto fragmenta los destinos entre un conjunto que sigue estudiando, otro que pasa a formar parte de la PEA, otro que busca compatibilizar trabajo y estudios, otro de los que no estudian ni trabajan, particularmente numerosos en los deciles con menores ingresos, donde el desempleo es alto, los empleos tienden a la descualificación y hay un segmento no menor de mujeres jóvenes inactivas que se dedican a los quehaceres del hogar, casi siempre asociado a una maternidad temprana que no hace más que revelar que los márgenes de libertad para las trayectorias posibles permanecen anclados a las posiciones heredadas por las generaciones precedentes -0 lo que Bourdieu define como trayectorias de clase.$-{ }^{18}$

\section{d) Calendarios de pareja y procreación}

La referencia a la maternidad temprana es importante por cuanto conduce el análisis hacia el cordón que permite transitar desde las trayectorias escolares y laborales al ámbito más «privado» de las biografías, o de la vida sexual y afectiva (Leccardi, 2006; Montilva, 2007, 2008). Aquí lo que nos interesa es ver algunas tendencias que permitan entender cómo se ha movido esa conexión. La primera refiere a la nupcialidad y sus calendarios. ${ }^{19}$ En el caso de las mujeres, lo que aparece es que durante toda la segunda mitad del siglo xx hubo dos tramos de edad que concentraron los casamientos: el de 20 a 24 años y el menor que 19 años. En 1952 los porcentajes de ambos conjuntos son relativamente similares, en 1960 casi empatan, pero desde 1970 las curvas se separan y hacia inicios de la década de los ochenta los matrimonios se concentran claramente entre los 20 y 24 años, aunque a partir de ahí esta curva también empieza un declive que contrasta con el ascenso en la cantidad de mujeres que se empieza a casar a los 25-29 o más años incluso. 


\section{Gráfico 6: porcentaje de casadas según tramo de edad sobre el total de matrimonios del año. Chile 1952-2010}

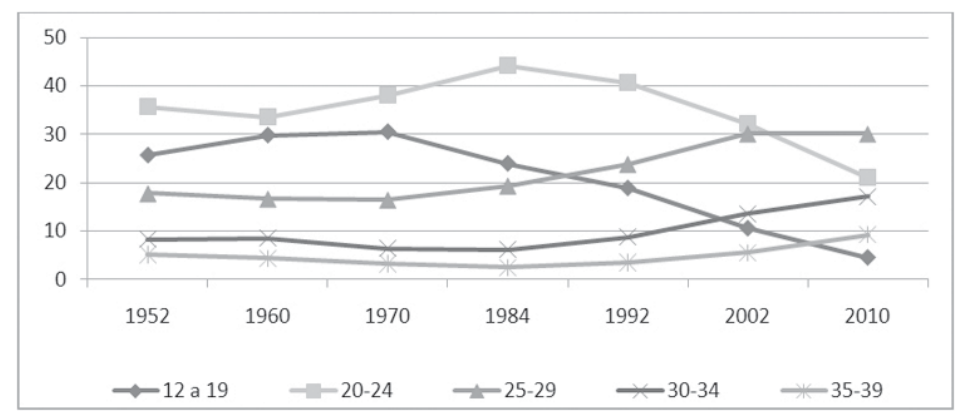

Fuente: Elaboración propia sobre la base de la serie de estadísticas vitales y demográficas delinE.

El resumen del período es que menos del 5\% de las mujeres que se casaron el 2010 eran menores de 20 años; el que había sido el tramo de edad históricamente «modal» — 20 a 24- dejó de serlo y, hacia el final del período, por primera vez el tramo entre 25 y 29 años concentra a la mayor parte de las casadas del año. La lectura es clara: las últimas generaciones de mujeres están llevando cada vez más lejos la tendencia a posponer —o simplemente desechar - el matrimonio como arreglo conyugal. De hecho, los únicos rangos de edades que siguen una tendencia al alza en su peso relativo en las últimas dos décadas son los posteriores a los 30 años.

En el caso de los hombres las tendencias son similares, aunque con algunos matices. El primero es que el matrimonio temprano - anterior a los 20 años - nunca fue alto y ahora es casi nulo. ${ }^{20} \mathrm{Y}$ el segundo es que las mismas tendencias se dan aquí más acentuadas, sobre todo en lo que respecta al aumento proporcional de los casamientos después de los 30 años, las únicas edades que en las últimas dos décadas suben —y rápido- en vez de bajar, al punto de representar a más de un tercio de los contrayentes en 2010. ${ }^{21}$ Lo importante es que en ambos sexos se produce este desplazamiento que convierte en «edades de soltería» las que fueran «edades para casarse» y en «edades para casarse» las que antes eran para "ya estar casados». Lo confirma el gráfico 7: las curvas muestran la evolución de los estados conyugales del tramo entre 25 y 29 años - «representativo» en tanto concentra la mayor parte de los y las contrayentes de nupcias en los últimos años- . Cada punto en las curvas indica la proporción de cada estado civil en relación a la población total de la cohorte respectiva. ${ }^{22}$ 


\section{Gráfico 7: tasa de nupcialidad según sexo y edad. Chile 1930-2011 23}

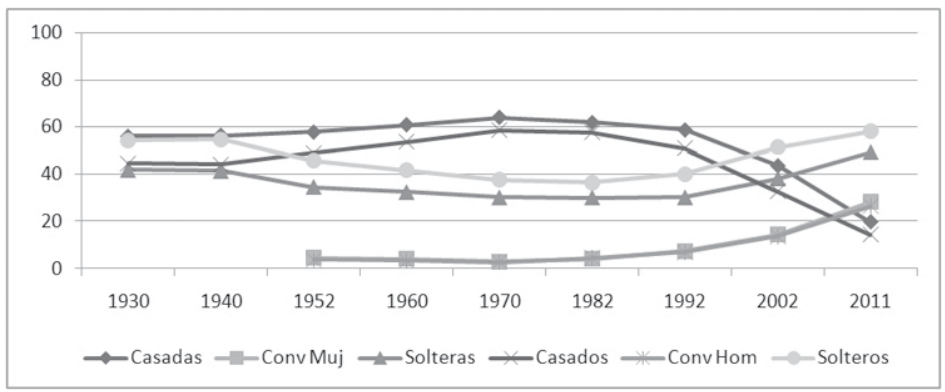

Fuente: Elaboración propia sobre la base de serie de censos de población 1952-2002, CASEN 2011.

El gráfico parte en 1930, cuando recién se empezaba a implementar una Política Social armada de dispositivos técnicos y un régimen de discurso que promovía a la familia nuclear bajo contrato matrimonial como modo normalizado de hogar y familia (Rosemblatt, 1995). ${ }^{24}$ Ya en la década de 1940 se notan sus primeros efectos, cuando bajen las personas solteras y comiencen a aumentar los casados, incluso entre los hombres, en una tendencia que va a continuar en las décadas siguientes hasta alcanzar su punto máximo en el censo de 1970.

Después de ese punto, el peso de los casados empieza un declive, al tiempo que aumentan, de a poco, los solteros y convivientes. El hecho que este quiebre coincida con el golpe militar sugiere una relación entre estas tendencias y el nuevo marco de políticas sociales, como si el desmantelamiento radical de las protecciones que extendía el Estado le hubiese quitado en parte el incentivo que llegó a tener el matrimonio. ${ }^{25} \mathrm{Y}$ si a eso se suman la inestabilidad laboral que trajo la flexibilidad neoliberal y la crisis del trabajo por desprotección y debilitamiento de la administración pública y la industria interna, se entiende que incluso formar una familia empezara a perder piso y condición de posibilidad para parte de las nuevas generaciones. ${ }^{26}$

Hasta 1992 el efecto aún es débil, pero de ahí en adelante toma fuerza y velocidad: los casados bajan fuerte, dejan de ser el estado civil más común a los 25-29 años y son desplazados primero por los solteros (2002) y luego incluso por los convivientes (2011), con la particularidad de que la aceleración de las últimas dos décadas expresaría la expansión de un nuevo marco cultural en que la institución del matrimonio, en formato civil y religioso, se ha vuelto un código sin fuerza regulatoria sobre la vida práctica. Primero porque las «normas del honor» y las prohibiciones de «la decencia» ya no amarran con la fuerza de antes la sexualidad y la independencia a la formalización de los vínculos de pareja; ${ }^{27}$ y segundo, porque la formación 
de pareja ahora se ciñe a la racionalidad práctica de «proyectos de vida» que buscan la individuación y eso implica, entre otras cosas, nuevos modos de resolver la compatibilidad entre actividad-pareja-procreación.

\section{e) Variaciones en el tiempo de los hijos}

Llegamos así al ámbito de la fecundidad. Aquí la pregunta es si ha habido cambios en la relación edad-fecundidad. La respuesta está en el gráfico 8, que informa sobre cómo se han movido las tasas específicas de fecundidad en los distintos tramos etarios. Lo que aparece es que la curva más antigua del gráfico (1952) indica una fecundidad a los 15-19 años que parte elevada, que alcanza su máximo entre los 20 y los 29 años y luego desciende en los tramos siguientes aunque a niveles altos si se los compara - en sentido vertical - con las curvas de años posteriores. ${ }^{28}$ La única curva que le supera es la de 1961, cuando ya no morían tantos niños al nacer ni mujeres al parir, ${ }^{29}$ y las políticas eugenésicas y «familistas» habían aumentado las probabilidades de embarazo y multiplicado el número de hijos por mujer a su máximo histórico (cf. Villalón y Vera, 2012).

\section{Gráfico 8: tasa específica de fecundidad según tramo etario. Chile 1950, 1961, 1979, 1993, 2009}

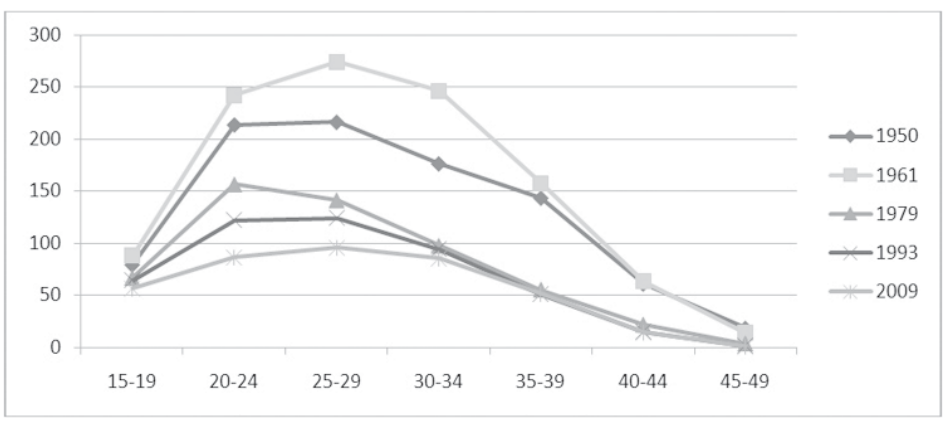

Fuente: Instituto Nacional de Estadísticas.

Pero ocurrió que mientras Chile entraba en esta fase avanzada de su primera transición demográfica y llegaba a ese peak de fecundidad, se habían empezado a activar una serie de políticas de regulación demográfica que buscaron evitar el embarazo no deseado, reducir las tasas de fecundidad y promover una «Paternidad Responsable» (Palma, 2000). En torno a esta biopolítica, diría Foucault (cf. Foucault, 1999), se alinearon organismos del Estado, onG's y agencias internacionales que van a hacer del control de la natalidad y la Planificación Familiar una Política de Estado. ${ }^{30}$ El resultado es que hacia fines de la década de 1970 ha pasado el baby-boom de los sesenta y la fecundidad está en declive en todas las edades. Las ciencias químicas habían logrado separar la menarquía de la fecundación y la sexua- 
lidad de la reproducción (Giddens, 1995), las mujeres empiezan a devenir «cyborgs» que borran con la química el límite entre naturaleza y ciencia (Haraway, 1991), y logran así ajustar los hijos tenidos a los deseados, que empiezan a ser menos y dejados para edades más tardías. Primero van a ser las «pioneras» del cambio reproductivo, mujeres urbanas, profesionales y de altos ingresos, como en México y otros países (Quilodrán y Juárez, op. cit.; Montilva, op. cit.); y luego serán los sectores «rezagados» en el control de la natalidad (Schkolnik y Chackiel, 1998) los que irán incorporando, a su modo, los nuevos métodos contraceptivos. ${ }^{31}$

Las curvas de 1979 y 1993 grafican este doble desplazamiento: hacia abajo y marcado, por la baja en la fecundidad en todas las edades; y hacia el lado, con máximos que avanzan hacia edades posteriores a los 25 años, aunque al mismo nivel que la tasa de fecundidad adolescente de 1961, tan baja que Chile hoy se ubica entre los países de América Latina con menor fecundidad junto a Cuba y Uruguay y ya aparecen voces alarmadas por el «problema» del reemplazo demográfico.

De nuevo el proceso es notable. La «distancia epocal» respecto a los años del baby-boom es sin duda considerable. Cada vez son más las mujeres que tienen su primer hijo a edades cercanas a los 30 o más años, y lo mismo y más se registra en el caso de los hombres. ${ }^{32}$ No obstante, en este aspecto de los cursos de vida vuelven a asomar los factores duros de la historia social bajo la forma, ahora, de diferencias en las tasas de fecundidad temprana y general (Rodríguez, 2005b). ${ }^{33}$ Esto porque sólo en los tres o cuatro deciles de más altos ingresos se puede encontrar un predominio de mujeres sin hijos a los 25 o más de 30 años; pero de los deciles intermedios para abajo, ya a los 20 años más de la mitad de las mujeres tiene 1, 2 o incluso 3 hijos. $^{34}$ Probablemente sea éste uno de los modos en que toman cuerpo las diferencias en los grados de integración a los espacios más avanzados del «mundo globalizado» y la adopción diferenciada o socialmente segmentada de los comportamientos reproductivos que caracterizan a la segunda transición demográfica. ${ }^{35} \mathrm{Y}$ lo interesante es que del recorrido que propone el texto nos encontramos con que i) existe una correspondencia entre los distintos ámbitos según la cual modificaciones en uno repercuten en el otro, ii) que esa correspondencia se debe a la unidad interna de cada biografía, y iii) que las diferencias en la forma en que se articulan estas unidades discretas resultan y son-expresión-de la acumulación de diferencias histórica en la distribución de los recursos para la construcción de biografías.

\section{A modo de cierre}

El propósito con este texto fue ilustrar cambios de época a partir de tendencias globales en los procesos biográficos de distintas generaciones. $\mathrm{Al}$ trabajar con datos censales asumimos que se nos escapan aspectos tan importantes como los efectos de la estratificación social y la subjetividad 
implicada en los procesos. Ese es el desafío pendiente. Para el cierre, sólo destacar algunos puntos que pueden quedar para una discusión.

a) El primero y central es la historicidad de los procesos biográficos. Los «tiempos biográficos» o las «etapas de la vida» se inscriben en coordenadas históricas que relativizan la relación entre edad cronológica y edad social. La diferencia se revela en la diacronía de las épocas: la variación en los pesos relativos de una misma situación —o categoría - a igual edad cronológica señala un cambio estructural en el modo de generación de los procesos biográficos. Se trata, en el fondo, del escenario que experimentó cada generación en su fase de tránsito-a-la-autonomía. Distintos en su diacronía por el desplazamiento cronológico de algunos eventos y tránsitos biográficos, mantienen, sin embargo, una continuidad justamente en la diferencia estructural-sincrónica de cada época particular que al final conecta la diacronía y sincronía.

b) Un segundo registro de esta historicidad de los procesos biográficos refiere al tramado de instituciones y actores de distinto tipo cuya evolución cada vez compone lo que Searle definiera como las condiciones «ontológicamente objetivas» y «subjetivas» para la acción (cf. Searle, 1990). En cada momento operaría lo que Agamben define como dispositivo, aquel complejo de prácticas-saberes-reglas-instituciones que buscan regular los espacios de acción (Agamben, 2011); y lo que Latour denomina colectivo (Latour, 2001), la red de actantes humanos y no-humanos que hacen lo social. ${ }^{36}$ Acaso sea ese uno de los desafíos para los estudios sobre cursos de vida: desplegar la genealogía de dispositivos y colectivos.

c) Hay un tercer registro de la historicidad que tiene un sentido, si se quiere, «toureniano», y que refiere a la acción de movimientos y actores que disputan la definición de los marcos culturales de una formación societal (cf. Touraine, 2000). Las tendencias en materia de escolaridad, trabajo, fecundidad no se podrían entender obviando, por ejemplo, la acción del movimiento feminista, que desde los años treinta hasta ahora viene desplazando el horizonte de vidas legítimas para las mujeres. Tampoco si pasa por alto la acción de los trabajadores organizados y su presión por sistemas de protección, o de los movimientos estudiantiles y su demanda por democratización del sistema educacional. Son todos sujetos colectivos que con su acción, no siempre continua ni estable, han modificado el campo para la producción de procesos biográficos.

d) Y en un cuarto registro está la historicidad de los sujetos individuales, que son, a fin de cuentas, los protagonistas de toda biografía. Ante el desafío de «hacer su propia historia», o lo que Martuccelli llama pruebas del proceso de individuación, ${ }^{37}$ los sujetos individuales son quienes deben leer las condiciones de su entorno y trazar desde ahí caminos, movilizar o no recursos, tomar decisiones o partido, dando forma con ello a la fuerza básica que actualiza el desplazamiento de las curvas — que no son sino la unión en un punto de un conjunto disperso de sujetos en acción-. 
e) El punto es que cuando más se nota esa historicidad es cuando las generaciones han entrado a su «fase» de activación. Fue este segmento en que se agudiza la pendiente de las curvas el que le dio forma a la «dinámica de las generaciones». Y la cuestión aquí es doble. Primero, que esa fase de cambios tiende a coincidir de forma cada vez más estrecha con el tiempo biográfico que va desde los 15 a los 25 años. ${ }^{38}$ Y segundo, que cualquiera fuera la curva, en los veinte años que van desde 1990 hasta el 2010, todos los ángulos se han agudizado en forma notoria. Probablemente sea lo acelerado de estos cambios una de las claves que subraya la dimensión política de los cursos de vida y que expresan, con fuerza y urgencia, los jóvenes contemporáneos. La razón es simple: es en este tiempo biográfico de activación cuando se está jugando, en buena medida, la fase posterior de «meseta». Pasado ese tiempo, la distribución social de «capitales» entre integrantes de una misma generación se estanca, definiendo, con ello, lo que será la estructura generacional de posiciones sociales. De ahí la centralidad que reviste toda acción política que apunte a remover los estratos de historia que siguen separando las posibilidades de acceso a recursos para la construcción de la propia historia.

f) Ese fue un punto común a los temas que tocó este texto. Las curvas vienen a ser un registro de la forma en que la «modernización», en sus distintas etapas, ha expandido el gobierno sobre los modos de vida. A grandes trazos, las décadas de 1930 a 1970 es cuando se instalan los dispositivos para cubrir las necesidades funcionales del proyecto desarrollista. En una segunda fase, que coincide, según lo visto, con la crisis política de principios de los setenta, las técnicas de gobierno de los grupos en el poder desmonta los soportes institucionales de la etapa anterior y obliga a la gestión individualizada del logro y el acceso a los recursos que antes proveyera el Estado. Hasta la década de los noventa se está todavía en una fase de instalación de la nueva matriz ideológica, pero desde los noventa en adelante la escala de los cambios. Sus efectos son ambivalentes. Por un lado, se acelera la descomposición de los atavíos de la «sociedad tradicional» y los medios difunden múltiples repertorios biográficos que pueblan los imaginarios de la «modernidad desbordada» (cf. Appadurai, 2001). Pero, por otro, instala una nueva forma de captura de los cuerpos y los tiempos que obliga a resolver tensiones hasta hace poco inexistentes, ${ }^{39}$ que sin embargo, pasan por los grados de integración a los sectores dinámicos de la nueva economía. Mientras los sectores privilegiados se apropian de las posiciones ventajosas de la economía financiero-exportadora y adoptan con rapidez los nuevos estilos de vida de los sectores globalizados, la «clase media», desfigurada como clase, se fragmenta en segmentos que entran a la competencia por títulos y puestos e incorporan los nuevos formatos biográficos y otros que quedan atrás aunque a cierta distancia de un mundo popular que, ante la clausura de los accesos, diseña sus propias estrategias de sobrevivencia y actualiza repertorios biográficos relativamente conocidos que mantienen la brecha de la desigualdad, pero también la distancia respecto al tipo de disciplinamiento que exige el modo de civilización actual. 
Estas sólo son hipótesis que requieren mayor investigación y que intentaremos profundizar con el análisis del material cualitativo para ver si hay elementos de sentido vinculados a pertenencias étnicas, convicciones religiosas u otros componentes identitarios que pudieran marcar diferencias relevantes. Por ahora, nos restringimos a esta primera lectura de tendencias. 


\section{Notas}

${ }^{1}$ Este artículo da cuenta de los resultados del proyecto de investigación Fondecyt №1120926: «Transiciones a la vida adulta: generaciones y cambio social en Chile»; y se ha beneficiado del Proyecto Anillo de Investigación en Ciencias Sociales «Juventudes: transformaciones socioeconómicas, sociopolíticas y socioculturales de los jóvenes en el Chile contemporáneo», Conicyt SOC 1108.

${ }^{2}$ Ryder define a las cohortes como conjuntos que nacen en determinado momento y crecen a un mismo tiempo y constituyen el «sustrato biológico» del cambio social, (Ryder, 1965), similar, en ese sentido, a las teorías sobre las generaciones como sujeto histórico de Ortega y Gasset o Karl Mannheim.

\section{${ }^{3}$ Respecto a los regímenes de transición, Fernández, Bonapelch y Anfitti, 2013.}

${ }^{4}$ Bourdieu utiliza el concepto Modos de generación para dar cuenta de las condicionantes sociales e históricas que determinan el proceso de posicionamiento de cada nueva generación en un determinado campo. Encuentra ahí la fuente de los cambios en la distribución de capitales y posiciones en un campo y de los llamados «conflictos intergeneracionales», que no tendrían que ver con la diferencia de edad o con procesos de orden psicológico, sino con las tensiones que genera la pugna por el posicionamiento entre los aspirantes y los posicionados-o el choque entre viejos y jóvenes-. Al respecto, de Pierre Bourdieu, La distinción. Para una descripción sintética, ver de Enrique Martín Criado, Producir la juventud.

${ }^{5}$ María Angélica Illanes relata cómo en una primera etapa fueron filántropos y policías quienes anduvieron convenciendo o incluso forzando a los niños y sus familias para que cambiaran el trabajo o la calle por la escuela de primeras letras (cf. Illanes, 1991).

${ }^{6}$ Es revelador, en ese sentido, que en los informes de los censos de la primera mitad del siglo xx la mayor parte de las páginas dedicadas a Educación estuviera dedicada a la medición del Alfabetismo.

${ }^{7}$ En Chile se denomina Liceo a los establecimientos de enseñanza secundaria y Escuela a los de enseñanza primaria o básica.

8 El concepto estrategias de reproducción de Bourdieu refiere, en lo fundamental, a la pregunta por el campo de oficios o profesiones en que invierten sus capitales los agentes, dónde «ponen sus fichas», lo que implica una evaluación de posibilidades mezclado con la historia de las inversiones familiares y las tendencias del «mercado de capitales», entre otros. Para mayor claridad, ver La distinción de Pierre Bourdieu.

${ }^{9}$ Emmanuel Barozet señala que los nuevos mecanismos de reproducción social que crea el Estado en la década de los años sesenta beneficiaron básicamente a los sectores medios y consolidaron la brecha con los sectores populares (Barozet, 2006).

${ }^{10}$ Para una síntesis de las políticas de educación durante los noventa en Chile, Cox, 2003.

${ }^{11}$ Como muestran los estudios sobre la Nueva Ruralidad y los Nuevos Territorios 
Agrarios, las transformaciones en las redes camineras y las tecnologías del transporte han reducido las distancias de muchas zonas antes aisladas y permiten flujos diarios a las ciudades y pueblos más cercanos que, entre otras cosas, facilitan el acceso a la educación de los habitantes rurales. Respecto a esto, Canales y Canales, 2013.

12 De acuerdo a la encuesta CASEN 2011, sólo en el decil de más altos ingresos se da que más del $40 \%$ de los jóvenes entre 15 a 19 años estudia en un establecimiento privado, que son el espacio de reproducción de las élites económicas y profesionales. En los sectores medios se reparten entre un sector que asiste a la educación privada con subvención del Estado (entre un 30\% y un 40\%), y otro segmento que asiste a establecimientos municipales (entre $25 \%$ a $40 \%$ ), que son donde se concentra la mayor parte de los estudiantes de los deciles con menores ingresos, en donde el 55\% al 60\% estudia a estos establecimientos y cerca del $30 \%$ lo hace en establecimientos subvencionados.

${ }^{13}$ Las primeras regulaciones para evitar que los niños y adolescentes se desempeñaran en «labores riesgosas» o que perjudicaran su salud, en Chile datan de principios de la década de 1920. Ya en 1921 el Estado había firmado los primeros acuerdos internacionales en la materia y desde ahí hasta el presente ha seguido adhiriendo a las normativas de Naciones Unidas, sin que desaparezca del todo el hecho en sí. De acuerdo a la Encuesta de Actividades de Niños, Niñas y Adolescentes (EANNA 2012), cerca de 230 mil niños y adolescentes realiza alguna actividad económica —el 6.9\% de esta población, y la mitad de ellos pertenece al quintil con menores ingresos.

14 La muestra quizás más evidente es que en los censos de 1930 y 1940 ni siquiera se los distinguió en el desglose de la PEA: usaron sólo dos categorías, de 15 a 19 años y de 20 años y más. Pasada esa edad se los asumía parte de la «gente que trabaja».

${ }^{15}$ El término adolescencia se formuló para designar la condición social y psicológica que posibilitó la expansión de la enseñanza media entre las élites de las sociedades desarrolladas (Erikson, 1993). La palabra «juventud», por su parte, cristalizó fuertemente asociada a la condición de estudiante universitario en la décadas del sesenta. Ambos conceptos han sido cuestionados desde diferentes ángulos por reduccionista y homogeneizante. De ahí, por ejemplo, que en los estudios sobre temas de juventud se haya propuesto pasar del concepto de juventud al de juventudes: de una primera noción que designa al sujeto-estudiantil-universitario de los sesenta a un sujeto juvenil social y culturalmente diverso en los noventa y dos mil. Al respecto ver Duarte, 2000.

${ }^{16}$ Los estudios que han tratado estos temas descubren una distancia generacional en términos de expectativas de realización entre las mujeres cuyo eje más fuerte radica en la importancia que tiene para las jóvenes actuales la educación como recurso para lograr sus metas (cf. Guzmán, Mauro y Araujo, 1999).

${ }^{17}$ Sobre esta oposición estructural Bourdieu articuló su argumento para sostener que la juventud no es más que una palabra (cf. Bourdieu, 1990).

${ }^{18}$ De acuerdo a la Encuesta CASEN 2011, en el caso de los hombres, se da que a los 2529 años, cuando en líneas gruesas la distribución de posiciones laborales está relativamente definida, cerca de un $35 \%$ a $40 \%$ de los jóvenes de los primeros tres deciles que trabajan lo hacen en empleos no cualificados, cerca de un $20 \%$ son operarios artesanales o de maquinaria más compleja, y sólo entre un $2 \%$ y un $5 \%$ son técnicos intermedios o profesionales y científicos. En los deciles de mayores ingresos, entre un $20 \%$ y un 
$45 \%$ son profesionales y científicos, cerca de un 13\%, técnicos intermedios, y entre un $9 \%$ y un $3 \%$ de trabajadores no calificados. En las demás edades se replica esta estructura.

${ }^{19}$ Para el análisis elaboramos un indicador que señala el porcentaje de personas que contrajo matrimonio a una edad específica respecto al total de personas que se casaron un año determinado. La fórmula sería: $\mathrm{X}=$ (n nupcias rango etario i/ $\mathrm{N}$ total nupcias)*100.

${ }^{20}$ Partió en un 5\% en 1952, en su punto máximo (1970) no superó el 10\% — cuando en las mujeres era $30 \%$ - y hoy es inferior al $1 \%$.

${ }^{21}$ Entre 1992 y 2010 los hombres que se casaron entre los 30 y 34 años pasaron de representar el $13 \%$ al $21 \%$; mientras que los que se casaron entre los 35 y 39 años, subieron del $5 \%$ al $12 \%$.

\section{${ }^{22}$ La fórmula es: $\mathrm{X}=(\mathrm{n}$ Estado Civil i/N cohorte)*100}

${ }^{23}$ El dato para el año 2011 es un estimado que se obtuvo de la Encuesta de Caracterización Socioeconómica Nacional (CASEN) de ese año.

${ }^{24}$ Herramientas clave para esta política fueron el Salario Familiar y la Asignación Familiar, dos medidas que privilegiaron largamente a las familias formalmente constituidas. Por eso operó como un incentivo para la formalización de las uniones que probablemente no habría tenido lugar sin estos dispositivos (Rosemblatt, 1995).

${ }^{25}$ Diversos estudios comparados han demostrado la relación entre sistemas de protección y cursos de vida (cf. Mayer, 2004; Mayer y Svallfors, 2005). Ahí la particularidad de Chile: estas tendencias lo harían un «caso» que confirma esta relación, aunque esta vez en un mismo país y no países distintos, como en los casos europeos.

${ }^{26}$ De algún modo la inestabilidad laboral y las altas tasas de desempleo de los años setenta y ochenta reeditaron los mismos límites para los proyectos de familia y emancipación que enfrentaron los jóvenes ante la destrucción de la economía campesina de mediados del siglo xix (Salazar, 2000). Quizá la diferencia esté en que la vida bajo un régimen totalitario impuso un orden de prioridades más apegado a la sobrevivencia, si es que no la resistencia, a los tiempos difíciles, que desplazó también a este tipo de compromisos.

${ }^{27}$ Se trata de códigos de origen colonial que resonaron todavía con relativa fuerza durante el siglo XX y que hoy se restringen a los grupos católicos y evangélicos renuentes al «cambio cultural». Antecedentes se encuentran en los estudios sobre la familia colonial en Chile y en los trabajos sobre la familia en la era de la modernización. Ver, por ejemplo, Salinas, 1994 y Valdés, 2007.

${ }^{28}$ Un dato resulta revelador: en 1952, cerca de un tercio de las jovencitas menores de 20 años que fueron madres ese año vieron nacer a su segundo, tercer o hasta cuarto hijo/a, y en algunos casos minoritarios, el quinto o más incluso (DGE, 1952).

${ }^{29}$ Respecto al tipo de parto, por ejemplo, si hacia 1930 y 1940 cerca del 40\% fueron partos sin asistencia, en l década del cincuenta bajaron a un tercio, y a partir de los años 
sesenta continuarían un descenso que hoy llega a un mínimo cercano al 5\% (INE, 1952, 1961, 2010).

${ }^{30}$ Como parte de los objetivos del Servicio Nacional de Salud (sNs), se crea en 1962 la Comisión Asesora en Política de Regulación de Fecundidad que, entre otras cosas, buscaba regular el número de embarazos para manejar la «epidemia» del aborto. De ahí saldrían las primeras políticas de planificación familiar y control de la natalidad de mediados de los sesenta implementadas en el marco de una alianza entre el Estado de Chile con corporaciones como APROFA y la Agencia Internacional para el Desarrollo de los Estados Unidos (USAID), que abrió la vía para distribuir «la píldora» a través del sistema público de salud (Palma, op. cit.).

31 Entre otros factores para este «rezago» estuvieron las diferencias en las posibilidades de acceso a los nuevos métodos anticonceptivos. Cuando recién llegaron a Chile, su compra exigió receta médica, algo vedado para las clases que no podían pagar la hora con el doctor. Hubo, sí, los programas pioneros que incorporaron con éxito su uso entre mujeres de sectores populares (nota 33), pero no resistieron a la dictadura y llegaron hasta ahí (Palma, op. cit.).

32 Según las estadísticas vitales, la proporción que fue padre en los años respectivos ha ido bajando en todos los tramos etarios, especialmente marcado en los tramos entre 25 y 34 años, que históricamente habían sido las edades a las que más frecuentemente se convertían en padre los hombres.

${ }^{33}$ En un estudio que comparó registros de los departamentos de neonatología de un hospital público - donde se atienden los sectores de menores recursos- y de una de las clínicas privadas más caras de Santiago se pudo comprobar la enorme diferencia en la composición etaria de las madres, con alta presencia de adolescentes en el hospital público y de mujeres de 30 y más años en la clínica privada. Al respecto, Fuentes etal., 2010.

${ }^{34}$ De acuerdo a datos de la encuesta CASEN 2011, en los primeros tres deciles, el porcentaje de madres entre 15-19 años llega al 13\%, mientras en los tres deciles superiores el porcentaje es 2,5\% a 4\%. Al pasar al tramo etario entre 20-24 años, los porcentajes de estos mismos deciles suben y se ubican entre el $10 \%$ y el $20 \%$, en su mayoría con un solo hijo, mientras en los tres deciles de menores ingresos se ubican en torno al 55\% y llegan a más del $80 \%$ en el tramo 25-29 años, y en los tres deciles superiores, se mueven entre el 50\% y más del 75\% la proporción de mujeres que todavía no son madres.

${ }^{35}$ La teoría de la segunda transición demográfica de Ron Lesthaeghe y Dirk van de Kaa, intenta dar cuenta de los procesos demográficos que caracterizan a las sociedades post-industriales: cambios en la fecundidad vinculada a la postergación del matrimonio, nuevos patrones en la conformación de pareja, entre otros procesos que son de orden sociocultural y ya no sólo de crecimiento económico y modernización. Al respecto ver Lestaeghe, 1995, 2010; Van de Kaa, 1987, 2001, 2002.

${ }^{36}$ Así, el debate sobre el «bien» de los niños y el deber del Estado se tradujo en una institucionalidad política que promulgó leyes sobre el trabajo infantil y financió sedes escolares que en la práctica modificaron la producción de los procesos biográficos. O las definiciones sobre el «rol natural» del hombre y la mujer, que se tradujeron en 
políticas sociales y eugenésicas imposibles de llevar a la práctica sin el trabajo de asistentes sociales, el avance de las ciencias, el concurso de médicos y una red de instituciones que se incorporan como actantes que terminaron interviniendo en la forma de las biografías. Y así también con los cambios en la organización del trabajo y los procesos productivos, las transformaciones en la infraestructura urbana y de los territorios, etc.

${ }^{37}$ Martuccelli se refiere así a los «desafíos históricos, socialmente producidos, culturalmente representados, desigualmente distribuidos, que los individuos están obligados a enfrentar en el seno de un proceso estructural de individuación» (Martuccelli, op. cit.:21).

${ }^{38}$ En efecto, no todos los eventos o «marcadores de tránsito a la adultez» se retrasan, como postulan algunos análisis. En líneas gruesas, varía según ámbito y sexo. El tránsito estudios/trabajo se retrasa en los hombres, pero entre las mujeres más bien se masifica, adelanta y acorta: lo que en generaciones ya mayores fue un paso dado por pocas mujeres y algunas a los 40 o más años, las generaciones actuales lo vuelven frecuente y a los 25 o 30 años ya está bien definido. Pero la formación de pareja y la procreación sí se postergan, y esto por parte de hombres y mujeres.

${ }^{39}$ Piénsese, por ejemplo, en los dilemas que plantea sobre los tiempos de las mujeres la actividad laboral y el cuidado de los hijos. O las tensiones que se genera entre la postergación de la maternidad y la degeneración cromosómica o el stock de óvulos. 


\section{Bibliografía}

Agamben, G. (2011), «¿Qué es un dispositivo?». Sociológica N²6. Universidad Autónoma de México, México.

Appadurai, A. (2001), La modernidad desbordada. Dimensiones culturales de la globalización. FCE, Buenos Aires.

Araujo, K., y D. Martuccelli (2012), Desafíos comunes. Retrato de la sociedad chilena y sus individuos. Lom, Santiago.

Arteaga, C., D. Sepúlveda y V. Araneda (2013), «Diversificación de las estructuras familiares: caracterización de las convivencias en Chile». Revista de Sociología $\mathrm{N}^{\circ} 27$. Universidad de Chile, Santiago.

Barozet, Emmanuelle (2006), «El valor histórico del pituto: clase media, integración y diferenciación social en Chile». Revista de Sociología N²0. Universidad de Chile, Santiago.

Beck, Ulrich (1998), La sociedad del riesgo: hacia una nueva modernidad. Paidós, Barcelona.

Beck-Gernsheim, E. (2003), La reinvención de la familia: En busca de nuevas formas de convivencia. Paidós, Barcelona.

Bertaux,D. (1999), «El enfoque biográfico: su validez metodológica, suspotencialidades». Proposiciones N²9. Ediciones sur, Santiago.

Binstock, G. y W. Cabella (2011), «La nupcialidad en el Cono Sur: evolución reciente en la formación de uniones en Argentina, Chile y Uruguay». En G. Binstock y J. Melo(coord..): Nupcialidad y familia en la América Latina actual. Asociación Latinoamericana de Población, Río de Janeiro.

Blanco, Mercedes (2011), «El enfoque del curso de vida: orígenes y desarrollo». Revista Latinoamericana de Población N8. ALAP, Buenos Aires.

Blanco, M. y E. Pacheco (2003), Trabajo y familia desde el enfoque del curso de vida: dos subcohortes de mujeres mexicanas. Universidad Autónoma del Estado de México, México.

Bordieu, Pierre (1988), La distinción. Criterios y bases sociales del gusto. Taurus, Madrid.

Ídem (1990), «La juventud no es más que una palabra». En: Sociología y cultura. Grijalbo, México.

Canales, Alejandro (2004), «Retos teóricos de la demografía en la sociedad contemporánea». Papeles de Población N40. Universidad Autónoma del Estado de México, México. 
Canales, AlejandroyManuel Canales (2013), «De la metropolización a las agrópolis: el nuevo poblamiento urbano en el Chile actual». Polis N³4. Universidad de Los Lagos, Santiago.

Casal, J., M. García, R. Merino y M. Quesada (2006), «Aportaciones teóricas y metodológicas a la sociología de la juventud desde la perspectiva de la transición». Papers. Revista de Sociología N79. Universidad Autónoma de Barcelona, Barcelona.

De Coninck, F. y F. Godard (1998), «El enfoque biográfico a prueba de interpretaciones: formas temporales de causalidad». En: Los usos de la historia de vida en las ciencias sociales. Anthropos, Barcelona.

Cox, C. (2003), «Las políticas educacionales de Chile en las últimas dos décadas del siglo xx». En: Políticas educacionales en el cambio de siglo. Editorial Universitaria, Santiago.

Davia, María (2013), «Factores determinantes en la decisión de tener el primer hijo en las mujeres españolas». Papeles de Población N75. Universidad Autónoma del Estado de México, México.

Deleuze, G. y P. Guattari (2004): Mil mesetas. Pre-textos, Barcelona.

Duarte, Claudio (2000), «¿Juventud o juventudes? Acerca de cómo mirar y remirar a las juventudes de nuestro continente». Última Década $\mathrm{N}^{\circ} 13$. Ediciones CIDPA, Valparaíso.

Erikson, Erik (1993), Sociedad y adolescencia. Siglo xxi, México.

Fernández, T., S. Bonapelch yV. Anfitti (2013), «Regímenes de transición al primer empleo: Chile, México, Estados Unidos y Uruguay comparados». Papeles de Población Nº76. Universidad Autónoma del Estado de México, México.

Kohli, M. (2005), «Le cours de vie comme institution sociale». En Enquête, Biographie et cycle devie. Disponible en: http://enquete.revues.org/ document78.html.

Ídem (2007), The Institutionalization of the Life Course: Looking Back to Look Ahead. European University Institute.

Ferrarotti, F. (2011), «Las historias de vida como método». Acta sociológica N56.

Foucault, Michael (2009), Nacimiento de la biopolítica: curso del Collège de France (1978-1979). Akal, Madrid.

Fuentes, A., C. Jesam, L. Devoto, B. Angarita, A. Galleguillos, A. Torres y A. Mackenna, A. (2010), «Postergación de la maternidad en Chile: una realidad oculta». Revista Médica de Chile, 138(10). 
Giddens, Anthony (1995), La transformación de la intimidad. Cátedra, Madrid.

Guichard, E., V. Concha, G. Henríquez, S. Cavalli y C. Lalive d’Epinay (2013), «Reconstrucción subjetiva del curso de la vida en Chile». Revista Mexicana de Sociología N75. Universidad Autónoma de México, México.

Güell, Pedro (2007), «La sociedad chilena ha cambiado de escala: ¿y ahora qué?». Temas de Desarrollo Humano Sustentable N¹3. PNUD, Santiago.

Güell, P., R. Frei y S. Palestini (2009), «El enfoque de las prácticas: un aporte a la teoría del desarrollo». Polis $\mathrm{N}^{\circ} 23$. Universidad de Los Lagos, Santiago.

Guzmán, V., A. Mauro y K. Araujo (1999), Trayectorias laborales de mujeres: cambios generacionales en el mercado de trabajo. Centro de Estudios de la Mujer, Santiago.

Haraway, Donna (1991), Ciencia, cyborgs y mujeres: la reinvención de la naturaleza. Cátedra, Madrid.

Henríquez, Helia y Verónica Uribe-Echeverría (2003), Trayectorias laborales: la certeza de la incertidumbre. Dirección del Trabajo, Santiago.

Illanes, M. A. (1991), «Ausente, señorita»: el niño chileno, la escuela para pobres y el auxilio, 1890-1990: hacia una historia social del siglo XX en Chile. Junta Nacional de Auxilio Escolar y Becas, Santiago.

Jacinto, C. (2006), «Estrategias sistémicas y subjetivas de transición laboral de los jóvenes en Argentina. El papel de los dispositivos de formación para el empleo». Revista de Educación N³41.

Latour, Bruno (2001), La esperanza de Pandora: ensayos sobre la realidad de los estudios de la ciencia. Gedisa, Madrid.

Leccardi, Carmen (2006), «Tiempo y construcción biográfica en la 'sociedad de la incertidumbre’: reflexiones sobre las mujeres jóvenes». Nómadas Nº16. Universidad Central, Bogotá.

Lesthaeghe, R. (1995), «The second demographic transition in Western countries: An interpretation». Gender and family change in industrialized countries.

Ídem (2010), «The unfolding story of the second demographic transition». Population and Development Review N³6.

Longo, María E. (2010), «Trayectorias laborales de jóvenes en Argentina». Tesis de Doctorado. Universidad de Buenos Aires. 
Martín-Criado, Enrique (1998), Producir la juventud: crítica de la sociología de la juventud. Istmo, Barcelona.

Martuccelli, Danilo (2010), «La individuación como macrosociología de la sociedad singularista». Persona y Sociedad, Vol. 24, N³. Universidad Alberto Hurtado, Santiago.

Mayer, K. (2004), «Whose lives? How history, societies, and institutions define and shape life courses». Research in Human Development $\mathrm{N}^{\circ} 1$.

Mayer, K. y S. Svallfors (2005), «Life courses and life chances in a comparative perspective». En: Analyzing inequality: Life chances and social mobility in comparative perspective.

Miret, P. (2005), «Pautas territoriales en la emancipación juvenil en España, cohortes de nacimiento 1924-1968». Papeles de Geografía N41. Universidad de Murcia, Murcia.

Montilva, Maira (2007), «Individualización femenina y cohabitación sin papeles: el caso de las profesionales de Santiago de Chile».

Ídem (2008), «Postergación de la maternidad de mujeres profesionales jóvenes en dos metrópolis latinoamericanas». Utopía y praxis latinoamericana: revista internacional de filosofía iberoamericana y teoría social $\mathrm{N}^{\circ} 41$.

Mora Salas, Minor yOrlandina de Oliveira (2009), «Los jóvenes en el inicio de la vida adulta: trayectorias, transiciones y subjetividades». Estudios Sociológicos, Vol. xxvII, N79. El Colegio de México, México.

OCDE (2004), Revisión de políticas nacionales de educación Chile. Ministerio de Educación, Santiago.

Oliveira, Orlandina y Brígida García (1990), «Trabajo, fecundidad y condición femenina en México». Estudios Demográficos y Urbanos N¹5. El Colegio de México, México.

Palma, Irma (2000), «Transformaciones de la fecundidad en la sociedad chilena». Disponible en www.pasa.cl.

Pacheco, E. y M. Blanco (2002), «En busca de la ‘metodología mixta’ entre un estudio de corte cualitativo y el seguimiento de una cohorte en una encuesta retrospectiva». Estudios Demográficos y Urbanos N 51 . El Colegio de México, México.

Ídem (2005), «Análisis del efecto edad-período-cohorte en el nivel de participación económica de tres cohortes de mujeres mexicanas». Papeles de Población N43.Universidad Autónoma del Estado de México, México.

Pérez-Baleón, Guadalupe (2012), «Desigualdades de género en el inicio de 
la vida laboral estable». Papeles de Población N72. Universidad Autónoma del Estado de México, México.

PNUD (2008), Desarrollo humano en Chile rural. Seis millones por nuevos caminos. PNUD, Santiago.

Pries, Ludger (1996), «¿Institucionalización o desinstitucionalización del curso de vida? Biografía y sociedad como un enfoque integrativo e interdisciplinario». Estudios Demográficos y Urbanos No32. El Colegio de México, México.

Quilodrán, J. y F. Juárez (2009), «Las pioneras del cambio reproductivo: un análisis partiendo desde sus propios relatos». Notas de Población N87. CELADE, Santiago.

Raczynski, D. (2001), Entrevistas a 39 personas de las regiones Metropolitana, Séptima y Novena que habían abandonado el sistema escolar entre los años 1997 y 2000. Asesorías para el Desarrollo, Santiago.

Roberti, M. (2012), «Rupturas y subjetividades: un acercamiento a la perspectiva de las trayectorias laborales». Trabajo y Sociedad $\mathrm{N}^{\circ} 18$. Universidad Nacional de Santiago del Estero, Santiago del Estero.

Rodríguez, Jorge (2005a), «Unión y cohabitación en América Latina: ¿modernidad, exclusión, diversidad?». Serie Población y Desarrollo N57. CELADE, Santiago.

Ídem (2005b), «Reproducción en la adolescencia». Revista de la CEPAL N86. CEPAL, Santiago.

Rojas, Jorge (1996), Los niños cristaleros: trabajo infantil en la industria. Chile, 1880-1950. DIBAM, Santiago.

Rosemblatt, Karin (1995), «Por un hogar bien constituido. El Estado y su política familiar en los Frentes Populares». En:Disciplina y desacato. Construcción de identidad en Chile, siglos XIX y XX. Ediciones sur, Santiago.

Ryder, Norman (1965), «The cohort as a concept in the study of social change». American Sociological Review, 843-861.

Salazar, Gabriel (2000), Labradores, peones y proletarios: formación y crisis de la sociedad popular chilena del sigloxix. LOM, Santiago.

Salinas, René (1994), «Uniones ilegítimas y desuniones legítimas: el matrimonio y la formación de la pareja en Chile colonial». En P. Gonzalbo yC. A. Rabell: La familia en el mundo iberoamericano. unAm, México.

Salinas, Viviana (2009), Matrimonio y convivencia a la luz de la Encuesta Bicentenario. Universidad Católica de Chile, Santiago. 
Polis, Revista Latinoamericana, Volumen 15, No 44, 2016

Scoppetta, O. (2009), «Cambios en las trayectorias de fecundidad masculina en Córdoba, Colombia». Papeles de Población N62. Universidad Autónoma del Estado de México, México.

Schkolnik, S. y J. Chackiel (1998), «América Latina: la transición demográfica en sectores rezagados». Notas de Población N²26. CELAdE, Santiago.

Searle, J. (1990), Actos de habla. Ensayo de filosofía del lenguaje. Cátedra, Madrid.

Sepúlveda, Leandro (2010), «Las trayectorias de vida y el análisis de curso de vida como fuentes de conocimiento y orientación de políticas sociales». Revista Perspectivas de Trabajo Social N² 21. Universidad Católica Cardenal Silva Henríquez, Santiago.

Schkolnik, Mariana (2006), «Trayectorias laborales de los jóvenes chilenos». En M. Charlín y J. Weller (comp.): Juventud y mercado laboral: brechas y barreras. FLACSO, Santiago.

Valdés, Ximena (2007), La vida en común: familia y vida privada en Chile y el medio rural en la segunda mitad del siglo XX. LOM, Santiago.

Van de Kaa, D. J. (1987), «Europe's second demographic transition». Population Bulletin $\mathrm{N}^{\circ} 42$.

Ídem (2001), «Postmodern fertility preferences: from changing value orientation to new behavior». Population and Development Review, 290-331.

Ídem (2002), «The idea of a second demographic transition in industrialized countries». Birth, 35, 45.

Van de Velde, C. (2005), «La entrada en la vida adulta. Una comparación europea». Revista de Estudios de Juventud N71. InJUve, Madrid.

Villalón, G. y S. Vera (2012), «Panorama demográfico en Chile contemporáneo: desafíos para la sociedad del siglo xxI». Anales de la Universidad de Chile $\mathrm{N}^{\circ}$ 3. Universidad de Chile, Santiago.

Thompson, P. (1993), «Historias de vida y análisis del cambio social». En Jorge Aceves (comp.), Historia oral. México. Instituto Mora/Universidad Autónoma Metropolitana.

Touraine, Alain (2000), Crítica de la modernidad. Fondo de Cultura Económica, México. 